\title{
Policy makers, advisers, and reputation
}

\author{
Phongthorn T. Wrasai and Otto H. Swank* \\ Erasmus University Rotterdam and Tinbergen Institute
}

12 March 2004

\begin{abstract}
When hiring an adviser (he), a policy maker (she) often faces the problem that she has incomplete information about his preferences. Some advisers are good, in the sense that their preferences are closely aligned to the policy maker's preferences, and some advisers are bad. Recently, some scholars have argued that the policy maker's power to replace her adviser induces the adviser to act more in line with the policy maker's interests. The idea is that the adviser's desire to put a stamp on future policy reduces his incentive to manipulate information. This paper shows that the policy maker's power to replace her adviser may harm her. The reason is that this power may have an adverse effect on the behavior of good advisers.
\end{abstract}

Keywords: Reputation, Signalling, Uncertainty, Policy decision-making JEL classification: D78, D82, D83

*Address of correspondence: Phongthorn T. Wrasai, Erasmus University Rotterdam, P.O. Box 1738, 3000 DR Rotterdam, Netherlands. E-mail: pwrasai@few.eur.nl. 


\section{Introduction}

The consequences of many policies are complicated and difficult to foresee. To reduce the chances of making wrong decisions, policy makers need information. Policy makers often lack time and expertise to collect information themselves. For this reason, they have to rely on others. A serious problem is that agents who have information about policy consequences are usually those who have a vested interest in the outcome (Milgrom and Robert, 1986). For example, it is very likely that army officers are much better informed about the pros and cons of alternative weapon systems than a policy maker. This does not always mean, however, that officers' recommendations concerning weapon systems are in the policy maker's interests.

Asymmetric information sometimes implies that those who have the formal authority to take decisions do not always actually take decisions (see Aghion and Tirole, 1997, on the distinction between formal and real authority). In the public choice literature, it is often argued that informed players, like government bureaucracies and interest groups, have too much influence on policy. An early contribution to this literature is Niskanen (1971), who argues that bureaucracies are too large, because bureaucrats are better informed than those who are supposed to oversee them (for a survey of this literature, see Mueller, 2003).

More recently, scholars have argued that the power of informed parties should not be exaggerated. One argument in this debate revolves around reputation. In general, policy makers and information providers do no meet once, but several times. An important implication is that the policy maker can punish information providers who have manipulated information. One obvious punishment is firing the adviser. Bendor et al. (2001, p. 256), for example, argues that "if the subordinate cheats (say by exploiting the discretion given to him), then the boss might retaliate by seizing control in the next period." Wittman (1995, p. 104-105) also emphasizes that the relationship between a policy maker (in his book, Congress) and the information

provider (the bureaucracy) should not be modeled as a one shot game. He argues that the power of the bureaucracy is limited, because their ongoing relationship allows the principal to act conditional on past outcomes. Lupia and McCubbins (1994, p. 105) also mention damage of reputation as a reason why an informed party may refrain from manipulating information. It is striking that Bendor et al. 
(2001), Lupia and McCubbins (1994) and Wittman (1995) all mention reputational concerns as a reason why information providers may act in line with the principal's interests, but that neither of them actually shows that this is actually the case. Or as Bendor et al. (2001) put it "The preceding was a "free" application of the theory of repeated games to delegation issues."

This paper analyzes a simple two-period model of a policy maker (she) and an adviser (he) to identify the conditions under which the policy maker's power to replace her adviser induces the adviser to act (more) in line with her interests. In each period, the policy maker makes a decision on a project, and an adviser gives a recommendation about the project. The policy maker has incomplete information about the adviser's preferences. There are good and bad advisers, in the sense that the preferences of good advisers are closer to those of the policy maker than the preferences of bad advisers. ${ }^{1}$. At the end of period 1, the policy maker can replace her adviser. This power creates reputational concerns. ${ }^{2}$ To put a stamp on future policy, an adviser wants to be re-appointed. We show that reputational concerns often induce bad advisers to act more in line with the policy maker's interests. This is the benefit of reputational concerns. However, reputational concerns may induce good advisers to act less in line with the policy maker's interests. This is a cost of reputational concerns. We show that the cost may exceed the benefit. It is even possible that reputational concerns hamper communication. Overall, our analysis shows that the policy maker's power to replace her adviser does not always help her to keep control of her adviser.

Our paper is related to the game-theoretical literature on building and maintaining a reputation. An early contribution to this literature is Kreps and Wilson (1982), who study how a long-run incumbent firm can build a reputation for playing tough against potential entrants. A key feature of their study is that the long-run firm can be tough or weak. In the same spirit, Persson and Tabellini (1990) describe how a central banker can build a reputation of always fighting inflation. They assume that agents have incomplete information about the weight the central banker gives

\footnotetext{
${ }^{1}$ Dur and Swank (forthcoming) show that the effort an adviser puts in collecting information depends on his preferences. This paper does not deal with information collection. Throughout, we assume that advisers possess information.

${ }^{2}$ Suurmond, Swank and Visser (forthcoming) analyze a model in which advisers differ in ability rather than preferences. In their model, an adviser's reputation refers to the probability that the adviser is able.
} 
to fighting inflation relative to boosting economic growth. We follow this literature in that incomplete information about an agent's preferences is an essential feature of our model.

In many studies on reputation effects, reputation is good for the long-run player. However, recently, Ely and Välimäki (2003) show that reputation can be bad. Important for this result is that reputational concerns may lead the good type long-run player to take an action that is harmful for both himself and the short-run player. The reason for this action is a fear of being perceived as a bad type. Reputation is bad if, in response to this action the short-run player decides not to participate. In our model, the behavior of the good type adviser also plays an essential role. His desire to put a stamp on future policy may induce him to act against the policy maker's interest. The policy maker, in turn may respond by ignoring her adviser's recommendation.

We depart from most literature on reputation effects in that we study a principalagent model. In Ely and Välimäki (2003), for example, the agents are a seller and buyers. These agents do not have a hierarchical relationship. An important feature of our model is that the principal can replace the agent. In this respect, our model builds on studies that analyze how well elections help voters to control office holders (see e.g. Barro, 1973, Ferejohn, 1986, and Persson, Roland and Tabellini, 1997). The basic insight these studies offer is that the possibility to send office holders home helps voters to control them. We show that this result does not carry over to a policy maker-adviser setting. More generally, we identify the conditions under which the policy maker benefits from having the power of replacing her adviser.

The rest of this article is organized as follows. Section 2 presents the model. Section 3 contains an analysis of a simple example. In Section 4, we analyze the more general model. Section 5 concludes.

\section{The model}

We consider a two-period model, $t=\{1,2\}$. In each period, a policy maker has to make a decision on a public project, $X_{t}$. There are two alternatives: the project is implemented, $X_{t}=1$ or the status quo is maintained, $X_{t}=0$. An implemented 
project yields a payoff to the policy maker equal to:

$$
U_{t}^{P}\left(X_{t}=1\right)=p+\mu_{t}
$$

The parameter $p$ denotes the policy maker's predisposition towards the project. Throughout, we assume that $p>0 .{ }^{3}$ The term $\mu_{t}$ reflects that the consequences of the project are uncertain. We assume that $\mu_{t}$ is drawn from a uniform distribution function with $\mu_{t} \in[-h, h]$. Moreover, we assume that $\mu_{1}$ and $\mu_{2}$ are independent of each other. We normalize by zero the payoff to the policy maker when she preserves the status quo $\left(U_{t}^{P}\left(X_{t}=0\right)=0\right)$. Clearly, if the policy maker could observe $\mu_{t}$, she prefers $X_{t}=1$ to $X_{t}=0$ if $\mu_{t}>-p$. However, we assume that the policy maker does not observe $\mu_{t}$. Since $p>0$, without further information about $\mu_{t}$, the policy maker chooses $X_{t}=1$. To ensure that our model describes an interesting situation, we assume that $p-h<0$. The implication is that without further information about $\mu_{t}$ the policy maker runs the risk of making a wrong decision on the project.

In each period the policy maker can hire one adviser. The hired adviser observes $\mu_{t}$. On the basis of the adviser's preferences, $a$, two types of advisers can be distinguished. The first type is relatively biased towards preserving the status quo. The preferences of advisers of this type are represented by:

$$
U_{t}^{\underline{a}}\left(X_{t}=1\right)=\underline{a}+\mu_{t}
$$

Advisers of the second type are relatively biased towards implementation:

$$
U_{t}^{\bar{a}}\left(X_{t}=1\right)=\bar{a}+\mu_{t}
$$

with $\bar{a} \geq \underline{a}$. By normalization, the payoff to any adviser equals zero when the policy maker preserves the status quo. Our model is a dynamic game of incomplete information. The policy maker does not know the adviser's type. The prior probability that $a=\underline{a}$ equals $\frac{1}{2}$. This prior is common knowledge. An adviser knows his own type. Throughout, we assume that the adviser who is relatively biased against implementation is the good adviser from the policy maker's point of view. That is, if the policy maker were able to observe $a$, she would choose an advisor with $a=\underline{a}$.

\footnotetext{
${ }^{3}$ The analysis of the case that $p<0$ is analogous.
} 
The hired adviser sends a message, $m_{t}$, about the project to the policy maker. This message is a recommendation. Two recommendations are possible: $m_{t} \in$ $\{Y, N\}$, with $m_{t}=Y$ denoting that the adviser recommends $X_{t}=1$, and $m_{t}=N$ denoting that the adviser recommends $X_{t}=0$. After the policy maker has received her adviser's message, she makes a decision about the project. An important feature of our model is that at the end of period 1, after the policy maker has received the adviser's message, the policy maker can replace her adviser. As in period 1, the probability that a new adviser's preferences are represented by $a=\underline{a}$ equals $\frac{1}{2}$. We assume that the replacement decision is made before outcomes are observed, in particular $\mu_{1}$. The description of the game is presented as below:

$$
<<\text { Insert Table } 1>>
$$

The equilibrium of our game is a set of strategies and posterior beliefs that satisfy the following conditions: ${ }^{4}$ (i) In each period, the decision made by the policy maker is optimal given her beliefs and given the strategies of the two types of advisers; (ii) In each period, the message sent by the adviser is optimal, given his type, and given the policy maker's strategy; and (iii) Beliefs are updated according to Bayes' rule.

\section{A simple case}

To illustrate why reputational concerns may hurt the policy maker, we start with analyzing a simple example. We assume that $\underline{a}=0$ and $\bar{a}>h$.

\section{Advice and policy in period 2}

Consider period 2. In period 2, the adviser has no incentive to build a reputation. Consequently, the bad adviser always recommends implementation, irrespective of $\mu_{2}$, and the good adviser recommends implementation if and only if $\mu_{2}>0$. Does the policy maker has an incentive to follow the adviser's recommendation? Suppose that $m_{2}=Y$. Clearly, if the policy maker knew that the good adviser had sent this message, it would be optimal for her to follow the adviser's recommendation:

\footnotetext{
${ }^{4}$ Our model is a simple cheap-talk game in the spirit of Crawford and Sobel (1982). It is wellknown that this type of model always has a pooling equilibrium. Our focus is on the identification of a separating equilibrium if such an equilibrium exists.
} 
$p+E\left(\mu_{2} \mid \mu_{2}>0\right)=p+\frac{1}{2} h>0$, since $p>0$. If the policy maker knew that the bad adviser had sent $m_{2}=Y$, implementation would yield a payoff equal to $p>0$. Hence, if $m_{2}=Y$, it is optimal for the policy maker to choose $X_{2}=1$. Now suppose that $m_{2}=N$. The policy maker infers from $m_{2}=N$ that the adviser is the good one, for a bad one would never send $m_{2}=N$. Ignoring the recommendation, that is choosing $X_{1}=1$, yields a payoff: $p+E\left(\mu_{2} \mid \mu_{2}<0\right)=p-\frac{1}{2} h$. Accordingly, if $p<\frac{1}{2} h$, it is optimal for the policy maker to follow advice. To put it differently, $p<\frac{1}{2} h$ is the condition for communication in period 2. As there are no reasons for building a reputation in period $2, p<\frac{1}{2} h$ would also be the condition for communication if our model were a static one. In the remainder of this subsection, we will assume that $p<\frac{1}{2} h$.

\section{The replacement decision}

At the end of period 1, the policy maker can replace her adviser. Below we will show that if $m_{1}=N$ in a communicative equilibrium, then the probability that the adviser is good is higher than the probability that the adviser is bad. In contrast, if $m_{1}=Y$, then the probability that the adviser is bad is higher than that he is good. A direct implication is that in a communicative equilibrium, the policy maker keeps her adviser if and only if $m_{1}=N$.

\section{Advice and policy in period 1}

Let us now analyze policy advice in period 1. Suppose that the policy maker will follow the adviser's recommendation. Later we will check whether it is optimal for the policy maker to do so. Consider a bad adviser. The bad adviser anticipates that if he sends $m_{1}=N$, he will be maintained as an adviser, while if he sends $m_{1}=Y$, he will be replaced. Sending $m_{1}=N$, thus guarantees that the project will be implemented in period 2. So, $m_{1}=N$ yields a payoff $\bar{a}$. Sending $m_{1}=Y$ implies that the adviser will be replaced. His payoff then equals $\bar{a}+\mu_{1}+\frac{1}{2} \bar{a}+\frac{1}{4}\left(\bar{a}+\frac{1}{2} h\right)$. It is easy to check that $m_{1}=Y$ yields a higher payoff than $m_{1}=N$ if:

$$
\mu_{1}>-\frac{1}{8} h-\frac{3}{4} \bar{a}
$$

Equation (1) implies that if $\bar{a}<\frac{7}{6} h$, then for some values of $\mu_{1}$ the bad adviser recommends against implementation. This is the benefit of reputational concerns. 
The desire to determine future policy induces a bad adviser to behave more in accordance with the policy maker's interest. If $\bar{a} \geq \frac{7}{6} h$, then reputational concerns never lead the bad adviser to recommend $X_{1}=0$. As in period 2 , the bad adviser then always recommends implementation.

Now consider the good adviser. Recommending implementation yields a payoff to the good adviser equal to $\mu_{1}+\frac{1}{8} h$. Instead, recommending status quo yields $\frac{1}{4} h$. Therefore, the good adviser recommends implementation if and only if $\mu_{1}>$ $\frac{1}{8} h$. Hence, reputational concerns induce a good adviser to recommend against implementation for a wider range of parameters. Since we assume that $p>0$, this is a cost of reputational concerns.

\section{The communication condition}

So far we have assumed that the policy maker follows the adviser's recommendation, that is communication takes place. Let us now determine under which conditions this assumption is warranted. Consider first the case that the bad adviser never recommends status quo, $\bar{a}>\frac{7}{6} h$. Then, conditional on $m_{1}=N$, the policy maker's payoff equals $p+\frac{1}{2}\left(\frac{1}{8} h-h\right)=p-\frac{7}{16} h$. The condition for communication requires that this expression is negative, implying $p<\frac{7}{16} h$. Recall that without reputational concerns the condition for communication is $p<\frac{1}{2} h$. Hence, in the case that $\bar{a}>\frac{7}{6} h$ the condition for communication is more restrictive with reputational concerns than without. The reason for this result is clear. Communication requires that $p+E\left(\mu_{1} \mid m_{1}=N\right)<0$. Reputational concerns have no effect on the behavior of a bad adviser and lead the good adviser to recommend status quo more frequently. So, $E\left(\mu_{1} \mid m_{1}=N\right)$ is higher with reputational concerns than without. Hence, the condition for communication becomes more restrictive. Now suppose that $\bar{a}<\frac{7}{6} h$. For example suppose that $\bar{a}=h$. From (1) we know that then the bad adviser recommends against implementation if $\mu_{1}<-\frac{7}{8} h$. The expected value of $\mu_{1}$, conditional on $m_{1}=N$ equals:

$$
E\left(\mu_{1} \mid m_{1}=N\right)=-\operatorname{Pr}\left(a=\bar{a} \mid m_{1}=N\right) \frac{15}{16} h-\operatorname{Pr}\left(a=\underline{a} \mid m_{1}=N\right) \frac{7}{16} h=-\frac{39}{80} h
$$

The implication is that the communication condition is satisfied if $p<\frac{39}{80} h$. Thus, also in this case the communication constraint is more restrictive with reputational concerns than without. 
Does the policy maker benefit?

Does the policy maker benefit from her power to replace her adviser in our current example? Above we have shown that in our example reputational concerns may jeopardize communication. It is evident that in case communication is hampered because of reputational concerns, reputational concerns make the policy maker worse off. Suppose that reputational concerns do not make communication impossible. In a static model (or in period 2), ex ante the policy maker's payoff would be:

$$
\frac{1}{2} p+\frac{1}{2} \frac{1}{2}\left(p+\frac{1}{2} h\right)=\frac{3}{4} p+\frac{1}{8} h
$$

If $\bar{a}>\frac{7}{6} h$, then the policy maker's payoff in period 1 equals:

$$
\frac{1}{2} p+\frac{1}{2} \frac{7}{16}\left(p+\frac{1}{2} \frac{9}{8} h\right)=\frac{23}{32} p+\frac{63}{512} h
$$

It is easy to verify that the expression in (2) exceeds the expression in (3). To understand why, recall that in case $\bar{a}>\frac{7}{6} h$, reputational concerns do not affect the behavior of the bad adviser and induce the good adviser to recommend status quo more often. Since $p>\underline{a}=0$, the good adviser recommends the status quo too frequently from the policy maker's point of view, even in the absence of reputational concerns. Reputational concerns thus make things worse. Thus, if $\bar{a}>\frac{7}{6} h$, then the power of the policy maker to replace her adviser does not discipline him. On the contrary, in expectations, recommendations are less in line with the policy maker's interest. Of course, an advantage of the power to replace the adviser is a higher probability that the adviser is good in period 2 .

Now suppose that $\underline{a}<\frac{7}{6} h$. Then, in period 1 , the policy maker's payoff equals:

$$
\frac{1}{2}\left(\frac{15}{16}\left(p+\frac{1}{2}\left(h-\frac{7}{8} h\right)\right)\right)+\frac{1}{2}\left(\frac{7}{16}\left(p+\frac{1}{2}\left(h+\frac{1}{8} h\right)\right)\right)=\frac{11}{16} p+\frac{39}{256} h
$$

A comparison between (2) and (4) shows that if $p>\frac{7}{16} h$, reputational concerns make the policy maker better off in period 1 . Now reputational concerns lead the bad adviser to behave more in line with the policy maker's interest. 


\section{The more general model}

We now turn to the more general case that $p>0, p<\bar{a}<h$, and $\underline{a}<\bar{a}$. Consider the second period. Just like in our simple example, in the more general case, the adviser has no incentive to maintain his reputation in period 2. When the condition for communication is satisfied, the good adviser therefore sends $m_{2}=Y$ if and only if $\mu_{2}>-\underline{a}$, and the bad adviser sends $m_{2}=Y$ if and only if $\mu_{2}>-\bar{a}$.

Is it optimal for the policy maker to follow the recommendation made by her adviser? First note that, since $p>0$, it is always optimal for the policy maker to follow advice if $m=Y$. So suppose $m_{2}=N$. Let $\theta$ denote the posterior probability that $a=\underline{a}$. If a new adviser is hired, then $\theta=\frac{1}{2}$; otherwise, $\theta$ depends on the outcomes of period 1 . It is easy check that the expected value of $\mu_{2}$, conditional on $m_{2}=N$, equals:

$$
\begin{aligned}
E\left(\mu_{2} \mid m_{2}=N\right) & =-\frac{(1-\theta)(h-\bar{a})}{h-\theta \underline{a}-(1-\theta) \bar{a}} \frac{1}{2}(h+\bar{a})-\frac{\theta(h-\underline{a})}{h-\theta \underline{a}-(1-\theta) \bar{a}} \frac{1}{2}(h+\underline{a}) \\
& =-\frac{h^{2}-\theta\left(\underline{a}^{2}-\bar{a}^{2}\right)-\bar{a}^{2}}{2(h-\theta \underline{a}-(1-\theta) \bar{a})}
\end{aligned}
$$

Communication requires that $p+E\left(\mu_{2} \mid m_{2}=N\right)<0$. A sufficient condition for

this inequality to hold is that $p-\frac{1}{2}(h+\underline{a})<0$. Hence, if it is optimal for the policy maker to follow the advice given by a good adviser, it is also optimal for the policy maker to follow the advice if she does not know the type of adviser. The reason for this result is that relative to a good adviser, a bad adviser is less likely to recommend $X_{2}=0$.

\subsection{Period 1}

At the end of the first period, the policy maker can replace her adviser. Below we will show that a bad adviser is more likely to send $m_{1}=Y$ than a good adviser. A direct implication is that the policy maker replaces her adviser if and only if $m_{1}=Y$.

Consider a bad adviser who observes $\mu_{1}$. Suppose that in period 1 the policy maker follows her adviser's recommendation. Then, sending $m_{1}=Y$ yields an 
expected payoff to the bad adviser equal to:

$$
\bar{a}+\mu+\frac{1}{2}\left[\frac{1}{4 h}(h+\bar{a})^{2}+\frac{1}{2 h}(h+\underline{a})\left(\bar{a}+\frac{1}{2}(h-\underline{a})\right)\right]
$$

The last term in (6) shows that by sending $m_{1}=Y$, the bad adviser anticipates that in period 2 policy will be based with probability $\frac{1}{2}$ on a good adviser's recommendation, and with probability $\frac{1}{2}$ on a bad adviser's recommendation. Sending $m_{1}=N$ yields an expected payoff to the bad adviser equal to:

$$
\frac{1}{4 h}(h+\bar{a})^{2}
$$

Straightforward algebra shows that (6) is greater than (7) if:

$$
\mu_{1}>\bar{u}=-\bar{a}+\frac{1}{8 h}(\bar{a}-\underline{a})^{2}
$$

Equation (8) gives the cutoff value of $\mu$ for a bad adviser. A bad adviser sends $m_{1}=Y$ if and only if $\mu>\bar{\mu}$. The last term of (8) reflects the benefits of reputational concerns. The adviser's desire for office induces him to recommend against the project for a wider range of $\mu$. The extent to which reputational concerns matter depends on the deviation of $\bar{a}$ from $\underline{a}$. The larger the deviation of $\bar{a}$ from $\underline{a}$, the higher is the cost of the appointment of $\underline{a}$ from $\bar{a}$ 's point of view.

Now consider a good adviser. Like the bad adviser, a good adviser anticipates that if he sends $m_{1}=Y$, he will not be reappointed. Analogous to the determination of $\bar{\mu}$, one can show that the good adviser sends $m_{1}=Y$ if and only if $\mu_{1}>\underline{\mu}$, where:

$$
\underline{\mu}=-\underline{a}+\frac{1}{8 h}(\bar{a}-\underline{a})^{2}
$$

Equation (9) implies that the desire to determine future policy also induces the good adviser to send $m_{2}=N$ for a wider range of parameters. Figure 1 below diagrammatically describes our situation.

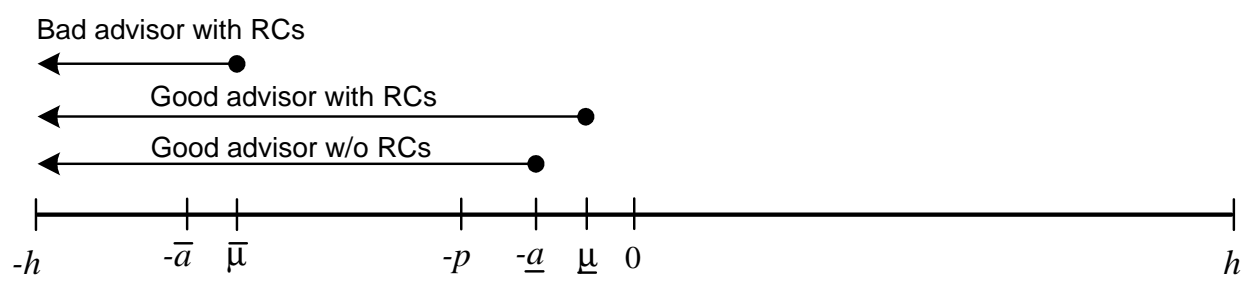


Figure 1: When does an advisor recommend against implementation in period 1?

The following Lemma summarizes the above discussion. ${ }^{5}$

Lemma 1 Suppose that the policy maker always follows the adviser's recommendation. Then, reputational concerns lead advisers to recommend against the project for a wider range of parameters.

So far, we have made two assumptions about the behavior of the policy maker. First, we have assumed that the policy maker always follows the adviser's recommendation. Second, we have assumed that the policy maker re-appoints the adviser if and only if $m_{1}=N$. Consider the re-appointment decision. The strategies of the two types of advisers imply the following posterior probabilities that the adviser is of the good type:

$$
\begin{aligned}
& \theta\left(m_{1}=Y\right)=\operatorname{Pr}\left(a=\underline{a} \mid m_{1}=Y\right)=\frac{h+\underline{a}}{2 h+\underline{a}+\bar{a}}<\frac{1}{2} \\
& \theta\left(m_{1}=N\right)=\operatorname{Pr}\left(a=\underline{a} \mid m_{1}=N\right)=\frac{h-\underline{a}}{2 h-\underline{a}-\bar{a}}>\frac{1}{2}
\end{aligned}
$$

Since the policy maker prefers a good adviser to a bad one, she strictly prefers to reappoint the adviser if $m_{1}=N$. Moreover, she prefers not to reappoint the adviser if $m_{1}=Y$. Hence, given the strategies of the two types of advisers discussed above, it is optimal for the policy maker to re-appoint her adviser if and only if $m_{1}=N$.

Let us now examine whether or not the policy maker has an incentive to follow an adviser's recommendation in period 1. Recall that because $p>0$, the policy maker always chooses $X_{1}=1$ if $m_{1}=Y$. Moreover recall that a sufficient condition for communication is that the policy maker follows the good adviser when he recommends against implementation.

Lemma 2 If $\underline{a} \geq p$, then the policy maker follows her adviser's recommendation.

Proof. Note that the larger is $\underline{\mu}$, the more restrictive is the condition for communication. Using (9), one can verify that the most restrictive case is $\underline{a}=p$ and

\footnotetext{
${ }^{5}$ An analogous lemma can be derived in case that the good adviser is biased towards implementation. Then, reputational concerns lead advisers to recommend implementation for a wider range of parameters.
} 
$\bar{a}=h$. If, in this case, the policy maker follows the good adviser, she will follow any recommendation if $\underline{a} \geq p$. For $\underline{a}=p$ and $\bar{a}=h$, the expected payoff to the policy maker, conditional on $m_{1}=N$, equals

$$
p-\frac{1}{2} h-\frac{1}{2} p+\frac{1}{16}(h-p)^{2}=\frac{1}{2}(h-p)\left[\frac{1}{8 h}(h-p)-1\right]
$$

Clearly, $0 \leq p<h$ ensures that the above expression is smaller than zero.

Lemma 2 presents a sufficient condition for which reputational concerns do not obstruct communication. We will now show that reputational concerns may raise a problem as to communication. If $m_{1}=N$, then the policy maker follows advice if $p+E\left(\mu_{1} \mid m_{1}=N\right)<0$. Using (8) and (9), it is easy to verify that:

$$
\begin{aligned}
E\left(\mu_{1} \mid m_{1}=N\right) & =\frac{h+\underline{\mu}}{h+\underline{\mu}+\bar{\mu}} \frac{1}{2}(\underline{\mu}-h)+\frac{h+\bar{\mu}}{h+\underline{\mu}+\bar{\mu}} \frac{1}{2}(\bar{\mu}-h) \\
& =-\frac{h^{2}-\frac{1}{2} \bar{\mu}^{2}-\frac{1}{2} \underline{\mu^{2}}}{h+\underline{\mu}+\bar{\mu}} \\
& =-\frac{h^{2}-\frac{1}{2}(z-\bar{a})^{2}-\frac{1}{2}(z-\underline{a})^{2}}{h-\underline{a}-\bar{a}+2 z} \text { with } z=\frac{1}{8 h}(\bar{a}-\underline{a})^{2}
\end{aligned}
$$

Differentiating (11) with respect to $z$ shows that $E\left(\mu_{1} \mid m_{1}=N\right)$ increases with $z$. The intuition behind this result is as follows. Reputational concerns induce both the good and the bad adviser to recommend against implementation for a wider range of $\mu_{1}$. A direct consequence is that the expected value of $\mu_{1}$, conditional on $m_{1}=N$, increases. Hence, if in the static model (or period 2), the condition for communication is just satisfied, reputational concerns may hamper communication. Note that $E\left(\mu_{1} \mid m_{1}=N\right)$ is independent of $p$. Hence, the higher is $p$ (for $p>0$ ), the more restrictive is the condition for communication, and the more likely it is that reputational concerns obstruct communication.

Proposition 1 For $\underline{a}<p$, reputational concerns may hamper communication.

We are now ready to evaluate the social consequences of reputational concerns. Using the optimal strategies of the two types of advisers, we can write the payoff to 
the policy maker in period 1 as:

$$
\begin{aligned}
& \operatorname{Pr}\left(m_{1}=Y\right)\left[p+\operatorname{Pr}\left(\bar{a} \mid m_{1}=Y\right) \frac{1}{2}(h-\bar{a}+z)+\operatorname{Pr}\left(\bar{a} \mid m_{1}=Y\right) \frac{1}{2}(h-\bar{a}+z)\right] \\
= & \frac{1}{4 h}(2 h+\bar{a}+\underline{a}-2 z) p+\frac{1}{4 h}\left[2 h^{2}-2 z^{2}-\bar{a}^{2}-\underline{a}^{2}+2 z(\bar{a}+\underline{a})\right]
\end{aligned}
$$

with $z=\frac{1}{8 h}(\bar{a}-\underline{a})^{2}$. Without reputational concerns, the policy maker's payoff would be equal to (12) with $z=0$. Hence, the policy maker benefits from reputational concerns if:

$$
z<\bar{a}+\underline{a}-p
$$

On the basis of (13), two cases can be distinguished. First, if $p>\underline{a}+\bar{a}$, then the right-hand side of $(10)$ is negative. Since, $z \geq 0$, the implication is that in this situation the policy maker suffers from reputational concerns. The reason is that if $p>\underline{a}+\bar{a}$, then without reputational concerns $X_{1}=0$ is recommended too frequently. Consequently, reputational concerns are bad, because they induce advisers to recommend $X_{1}=0$ even more frequently. Second, if $p<\underline{a}+\bar{a}$, then the opposite situation occurs. Without reputational concerns, $X_{1}=1$ is recommended too often. If $z$ is not too large, then the policy maker benefits from reputational concerns. The next proposition summarizes the above discussion:

Proposition 2 If (i) reputational concerns hamper communication or (ii) $p \geq \underline{a}+$ $\bar{a}$, then the policy maker's power to replace her adviser does not give her a higher payoff in period 1.

\section{Conclusions}

We have analyzed a simple two-period model of a policy maker and an adviser to show that the policy maker's power to replace her adviser may harm the policy maker. On the one hand, the fear of being replaced induces a bad adviser to act more in line with the policy maker's interests. On the other hand, the policy maker's power to replace her adviser may lead a good adviser to act less in line with the policy maker's interests. We show that the latter effect may dominate the former. Moreover, the latter effect may induce the policy maker to ignore policy advice. When reputational concerns are bad, the policy maker benefits from committing 
herself to always keeping her adviser.

Our results are derived from a model that is based on several restrictive assumptions. Let us briefly discuss two of them. One important assumption is that the policy maker could only consult one adviser. If the policy maker were able to consult more advisers, a comparison of the various recommendations could reveal information about the advisers' types. This may have important qualitative implications for our results. Second, in our model information collection is exogenous. Dur and Swank (forthcoming) show that advisers who are biased neither towards status quo nor towards implementation put most effort in collecting information. We conjecture that reputation effects weaken an adviser's incentive to collect information. The reason is that the desire to put a stamp on future policy induces an adviser to make less use of information. This reduces the benefits of information, and in turn leads an adviser to put less effort in collecting information.

\section{References}

[1] Aghion, P. and Tirole, J. (1997). Formal and real authority in organizations. Journal of Political Economy 105: 1-29.

[2] Barro, R.J. (1973). The Control of politicians: an economic model. Public Choice 14:19-42.

[3] Bendor, J., Glazer, A., and Hammond, T. (2001). Theories of delegation in political science. Annual Review of Political Science Vol. 4: 235-269.

[4] Crawford, V.P. and Sobel, J. (1982). Strategic information transmission. Econometrica 50: 1431-1451.

[5] Dur, R.A.J. and Swank, O.H. (forthcoming). Producing and manipulating information. Economic Journal.

[6] Ely, J.C. and Välimäki, J. (2003). Bad reputation. Quarterly Journal of Economics 118, Issue 3: 785-814.

[7] Ferejohn, J.A. (1986). Incumbent performance and electoral control. Public Choice, 50: 5-26. 
[8] Kreps, D.M. and Wilson, R.B. (1982). Reputation and imperfect information. Journal of Economic Theory 27: 253-279.

[9] Lupia, A. and McCubbins, M.D. (1994). Designing bureaucratic accountability, Law and Contemporary Problems, 57: 91-126.

[10] Milgrom, P.R. and Roberts, J. (1986). Relying on the information of interested parties. Rand Journal of Economics 17: 18-32.

[11] Mueller, D.C. (2003). Public Choice III, Cambridge: Cambridge University Press.

[12] Niskanen, W.A. (1971). Bureaucracy and Representative Government. Chicago: Aldine-Atherton.

[13] Persson, T. and Tabellini, G. (1990). Macroeconomic policy, credibility and politics. Harwood Academic Publishers, Chur, Switzerland.

[14] Persson, T., Roland, G., and Tabellini, G. (1997). Separation of powers and political accountability. Quarterly Journal of Economics 112: 1163-1202

[15] Suurmond, G., Swank, O.H. and Visser, B. (forthcoming). On the bad reputation of reputational concerns, Journal of Public Economics.

[16] Wittman, D. A. (1995). The myth of democratic failure: why political institutions are efficient, University of Chicago Press, Chicago. 
Table 1: The description of the game

Players: The policy maker $P$ and an advisor $A$

Period 1

- Nature chooses $\mu_{1} \in[-h, h]$ and $a \in\{\underline{a}, \bar{a}\}$.

- $A$ observes $\mu_{1}$, and sends a message $m_{1} \in\{Y, N\}$.

- $P$ observes $m_{1}$ and chooses between $X_{1}=0$ and $X_{1}=1$.

- $P$ chooses whether to keep her current advisor or to replace him.

Period 2

- Nature chooses $\mu_{2} \in[-h, h]$, and if the adviser of period 1 is replaced, $a \in$ $\{\underline{a}, \bar{a}\}$.

- $A$ observes $\mu_{2} \in[-h, h]$, and sends a message $m_{2} \in\{Y, N\}$ to $P$.

- $P$ observes $m_{2}$ and chooses between $X_{1}=0$ and $X_{1}=1$.

- Payoffs are realized.

Payoffs:

$U^{P}\left(X_{t}=1 \mid \mu_{t}\right)=p+\mu_{t}$ and $U^{P}\left(X_{t}=0\right)=0$. where $t=1,2$.

$U^{a}\left(X_{t}=1 \mid \mu_{t}\right)=a+\mu_{t}$ and $U^{a}\left(X_{t}=0\right)=0 ; a \in\{\underline{a}, \bar{a}\}$. 